\title{
QCD Studies with W and Z Measurements at LHC
}

\section{Imai JEN-LA PLANTE* for the ATLAS and CMS Collaborations}

Enrico Fermi Institute, University of Chicago

E-mail: imai@uchicago.edu

Events containing $\mathrm{W}$ or $\mathrm{Z}$ bosons, with subsequent leptonic decays, are important channels to test the Standard Model. They will be copiously produced at the Large Hadron Collider (LHC). Identification of these events will probe QCD predictions for the associated production of $\mathrm{W}$ or $\mathrm{Z}$ bosons with one or more jets. In addition, differential measurements such as the $\mathrm{W}$ charge asymmetry and the $\mathrm{Z}$ rapidity distribution can constrain the parton distribution functions of the proton. Studies of the prospects for these measurements using the ATLAS and CMS detectors are presented, with emphasis on the potential for the first years of LHC operation.

European Physical Society Europhysics Conference on High Energy Physics

July 16-22, 2009

Krakow, Poland

${ }^{*}$ Speaker. 


\section{Introduction}

The ATLAS [1] and CMS [2] detectors are installed, commissioned, and ready for data-taking with the first collisions from the LHC. Both general-purpose detectors will have access to a wide range of measurements including new physics searches. Their physics programs will require the validation of Standard Model (SM) theoretical predictions at collision energies much higher than any previously explored.

A key component of this effort will be measurements of the $\mathrm{W}$ and $\mathrm{Z}$ bosons, selected by their leptonic decays to electrons and muons. Relatively large cross-sections and the presence of high transverse momentum $\left(p_{T}\right)$ leptons; jets; and, in the case of the $\mathrm{W}$, missing transverse energy (MET) make these important backgrounds to SM processes such as top quark production and many new physics models. The rich event topologies will exercise the combined performance of the detector subsystems. These benchmark channels will also provide an opportunity to study QCD through measurements of the cross-sections for $\mathrm{W} / \mathrm{Z}+\mathrm{jets}$ and to constrain the parton distribution functions of the proton (PDFs) with differential $\mathrm{W}$ and $\mathrm{Z}$ measurements.

\section{2. $\mathrm{W} / \mathrm{Z}+$ jets cross-section measurements}

Both experiments will measure the cross-sections for the associated production of $\mathrm{W}$ or $\mathrm{Z}$ bosons with jets. The results will probe next-to-leading order (NLO) perturbative QCD calculations, currently available for up to $\mathrm{W}+3$ or $\mathrm{Z}+2$ jets. ATLAS has examined the prospects in detail, focusing on $\mathrm{Z}+\mathrm{jets}$ as a prototype and assuming an integrated luminosity of $1 \mathrm{fb}^{-1}$ at a collision energy of $\sqrt{s}=14 \mathrm{TeV}$ [3].

Events are triggered by requiring one or two isolated leptons. Offline selection is then applied, consisting of lepton pseudorapidity $(\eta)$ cuts and a lepton $p_{T}$ threshold, as well as calorimeter shower shape and tracking requirements in the case of the electrons. In addition, the invariant mass of the $\mathrm{Z}$ candidate is required to be near the $\mathrm{Z}$ mass resonance. For the $\mathrm{W}$ selection, this is replaced by a MET threshold. These criteria and their expected performance are detailed in [3].

For this study, a seeded cone algorithm with radius 0.4 is used to reconstruct the jets. Corrections for jet reconstruction efficiency, scale non-linearities, and resolution are derived from fully simulated Monte Carlo samples and applied to obtain results at hadron level. Jets with an angular separation $(\Delta \mathrm{R})$ less than 0.4 from the leptons are rejected. Alternative infrared and collinear safe jet reconstruction algorithms are currently being considered.

The relative contributions of the SM backgrounds vary with jet multiplicity. Top pair production is the dominant background at high multiplicity, while QCD multi-jet events with one or two jets mis-identified as leptons are more significant at low multiplicity. The large jet production crosssection combined with the low lepton fake rate makes it difficult to model the QCD background from simulation, so data-driven techniques must be used. An uncertainty of $20 \%$ is assumed on the QCD multi-jet background fraction estimated using Monte Carlo.

Figure 1 shows the expected results for $\mathrm{Z} \rightarrow$ ee+jets, obtained with fully simulated Monte Carlo pseudo-data generated with ALPGEN interfaced with Herwig for the parton showers. The uncertainties in Fig. 1(a) are expressed as a fraction of the ratio of the measured cross-sections relative to theoretical predictions, assuming a ratio of 1 . The dominant theoretical uncertainty comes from 
the PDFs, and it can be reduced by additional constraints from differential $\mathrm{W}$ and $\mathrm{Z}$ measurements as discussed in the next section. The effect of jet energy scale (JES) uncertainty is shown for both 5 and $10 \%$ JES uncertainty estimates. This uncertainty is expected to decrease with in situ measurements such as dijet, $\gamma+$ jet, and Z+jet balance, toward the design goal of $1 \%$. In Fig. 1(b), the comparison with predictions from the MCFM [4] program, corrected to hadron level, illustrates the sensitivity to differences between LO and NLO predictions.

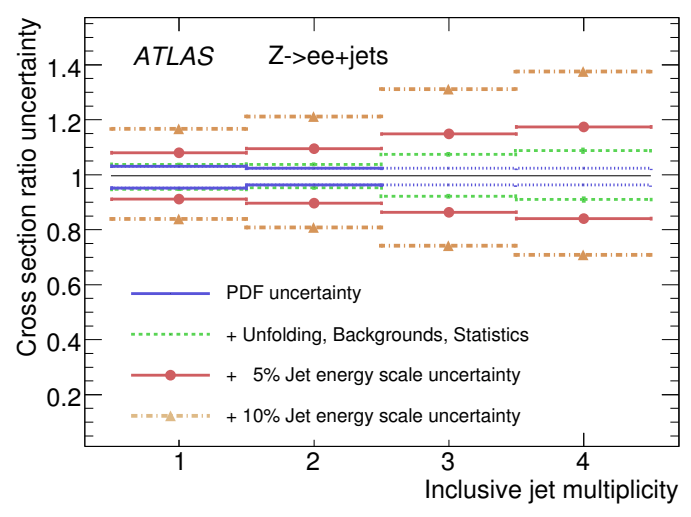

(a) Uncertainties.

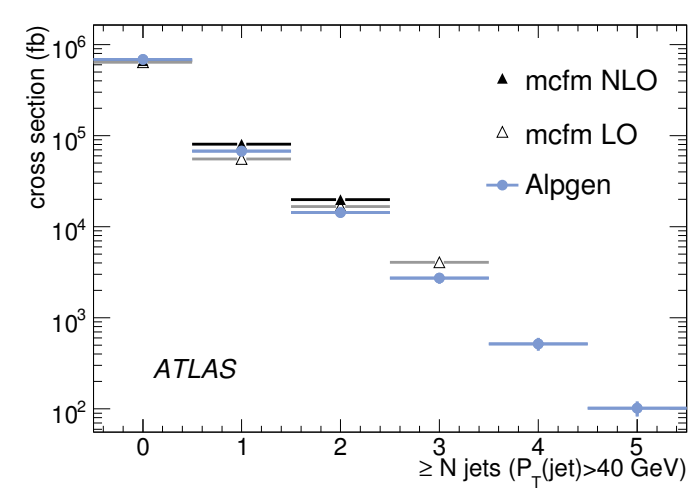

(b) Hadron level comparison of pseudo-data with theory.

Figure 1: Expected $\mathrm{Z} \rightarrow \mathrm{ee}+\mathrm{jets}$ cross-section results with $1 \mathrm{fb}^{-1}$ of data at $\sqrt{s}=14 \mathrm{TeV}$.

\section{Differential measurements}

\subsection{W charge asymmetry}

More $\mathrm{W}^{+}$than $\mathrm{W}^{-}$production is expected at the LHC due to the excess of $\mathrm{u}$ valence quarks relative to $\mathrm{d}$ in the proton. The charge asymmetry

$$
A(\eta)=\frac{\frac{d \sigma}{d \eta}\left(W^{+} \rightarrow \mu^{+} v\right)-\frac{d \sigma}{d \eta}\left(W^{-} \rightarrow \mu^{-} v\right)}{\frac{d \sigma}{d \eta}\left(W^{+} \rightarrow \mu^{+} v\right)+\frac{d \sigma}{d \eta}\left(W^{-} \rightarrow \mu^{-} v\right)}
$$

can be measured as a function of muon pseudorapidity, which is correlated to the boson rapidity. The predicted shape is sensitive to PDF modeling. CMS expects this measurement to have uncertainties competitive with current PDF uncertainties using $100 \mathrm{pb}^{-1}$ of data at $\sqrt{s}=10 \mathrm{TeV}$ [5].

Subtraction of the QCD background is again one of the primary experimental challenges, and a data-driven approach has been developed. Figure 2 compares the distribution of $\mathrm{W} \rightarrow \mu v$ signal (left) and QCD background (right) events as a function of MET and an isolation variable $\left(z=1-\frac{p_{T}}{p_{T}+I s o}\right)$ where $p_{T}$ is the muon transverse momentum and Iso is the sum of transverse energy deposits in the calorimeter within a cone of radius 0.3 around the muon direction, minus the muon contribution. Assuming the two variables are uncorrelated, the number of background events in the signal region $(z<0.05$ and MET $>20 \mathrm{GeV})$ can be estimated from the number of events in the sidebands.

Figure 3 shows the expected results, with a statistical uncertainty of $\sim 0.5 \%$. The systematic uncertainty is dominated by the precision of the assumed trigger and offline efficiency ratio of 1, obtained from Drell-Yan Monte Carlo. The expected total uncertainty is sufficiently small to constrain the PDF uncertainty, indicated by the yellow band. 

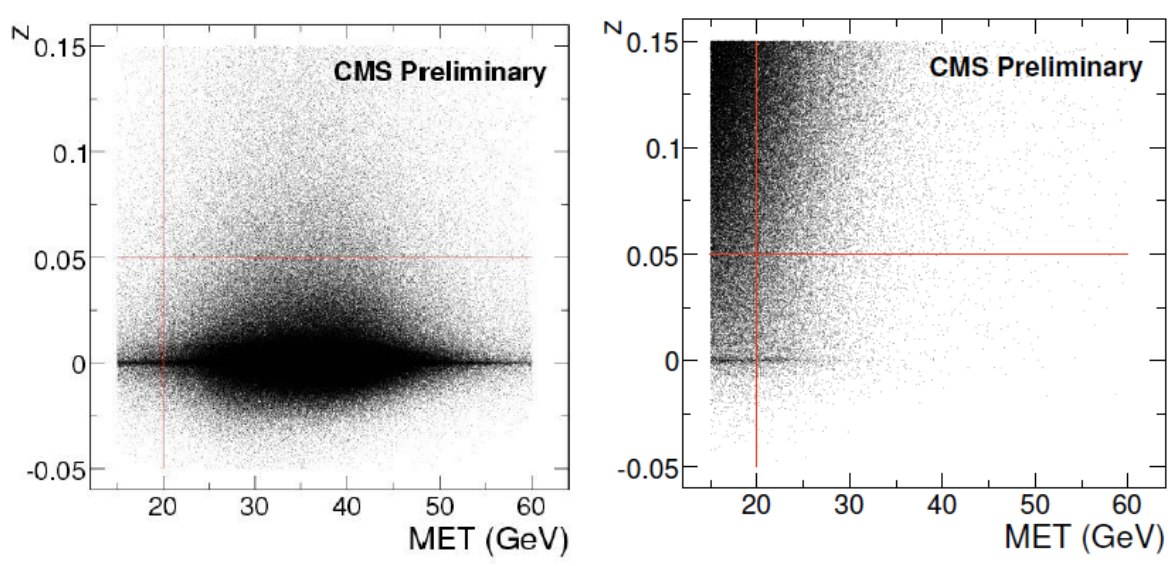

Figure 2: Distribution of $\mathrm{W} \rightarrow \mu v$ signal (left) and QCD background (right) events as a function of MET and $z$ (see text), showing the signal region with $z<0.05$ and MET $>20 \mathrm{GeV}$. The population at $z=0$ is increased by events with low measured isolation energy, due to zero-suppressed readout in the CMS calorimeters [2].

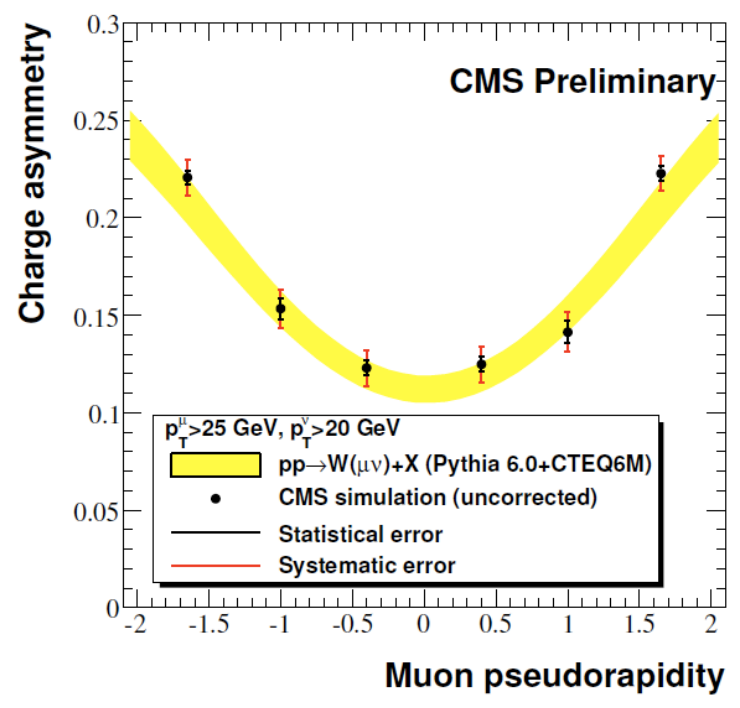

Figure 3: Expected $\mathrm{W} \rightarrow \mu v$ charge asymmetry results with $100 \mathrm{pb}^{-1}$ of data at $\sqrt{s}=10 \mathrm{TeV}$. The PDF uncertainty (yellow band) was obtained by reweighting.

\section{2 $\mathrm{Z}$ rapidity}

The shape of the $\mathrm{Z}$ boson rapidity distribution provides another probe of the PDFs. It has been computed up to next-to-next-to-leading order with low theoretical uncertainty, aside from the PDF evolution [6]. CMS has prepared a prototype measurement of this shape in the $\mathrm{Z} \rightarrow \mathrm{e}^{+} \mathrm{e}^{-}$channel, assuming $100 \mathrm{pb}^{-1}$ of data at $\sqrt{s}=10 \mathrm{TeV}$ [7].

The measurement relies on the bin-by-bin determination of the total efficiency to trigger, reconstruct, and identify the $\mathrm{Z}$ boson, as a function of rapidity $\left(y_{z} \equiv \frac{1}{2} \ln \frac{E+p_{L}}{E-p_{L}}\right)$, where $p_{L}$ is the longitudinal momentum of the $\mathrm{Z}$. Monte Carlo convolution is used to calculate this efficiency from single electron efficiencies, which will be measured in situ using a tag-and-probe method. 
Decays with one electron beyond the tracker acceptance are reconstructed using the forward hadron calorimeter to identify electrons with $3.1<|\eta|<4$.6. This extends the coverage up to $\mathrm{Z}$ rapidity of about 3.5, as demonstrated in Fig. 4. With the expected precision, this measurement will provide an early constraint of the PDFs.

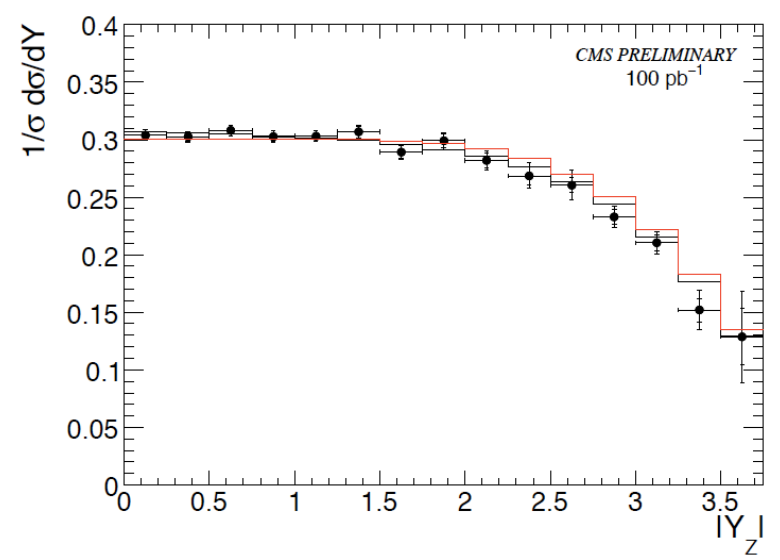

Figure 4: Expected precision of the $\mathrm{Z} \rightarrow$ ee rapidity distribution measured with $100 \mathrm{pb}^{-1}$ of data at $\sqrt{s}=10$ $\mathrm{TeV}$. The predicted shape is shown using CTEQ6.5 PDFs (black line) and for a positive variation of basis vector \#13 (red line), one of the basis vectors with the largest sensitivity to this measurement.

\section{Conclusions}

The potential for QCD studies with the measurements of $\mathrm{W}+\mathrm{jets}$ and $\mathrm{Z}+\mathrm{jets}$ cross-sections, the $\mathrm{W}$ charge asymmetry as a function of lepton pseudorapidity, and the shape of the $\mathrm{Z}$ rapidity distribution have been analyzed by ATLAS and CMS. Using early data, these measurements can improve our understanding of the PDF uncertainties and other QCD predictions.

\section{References}

[1] ATLAS Collaboration, G. Aad et al., The ATLAS Experiment at the CERN Large Hadron Collider, JINST 3 (2008) S08003

[2] CMS Collaboration, The CMS Experiment at the CERN LHC, JINST 3 (2008) S08004

[3] ATLAS Collaboration, G. Aad et al., Expected Performance of the ATLAS Experiment : Detector, Trigger and Physics, CERN-OPEN-2008-020 [hep-ex/0901.0512]

[4] J.Campbell, R.K.Ellis, Next-to-leading order corrections to $W+2$ jet and $Z+2$ jet production at hadron colliders, Phys. Rev. D 65 (2002) 113007 [hep-ph/0202176]; http://mcfm.fnal.gov/

[5] CMS Collaboration, Muon differential cross section and charge asymmetry in inclusive $p p \rightarrow W(\mu v)$ + X production at $\sqrt{s}=10 \mathrm{TeV}$, CMS PAS-EWK-09-003

[6] C. Anastasiou, et al., High-precision QCD at hadron colliders: Electroweak gauge boson rapidity distributions at next-to-next-to leading order, Phys. Rev. D 69 (2004) 094008 [hep-ph/0312266v2]

[7] CMS Collaboration, Study of the $Z \rightarrow e$ differential cross section as a function of $Z$ rapidity at $\sqrt{s}=$ $10 \mathrm{TeV}$, CMS PAS-EWK-09-005 